\title{
Proliferation of information - the good the bad and the ugly
}

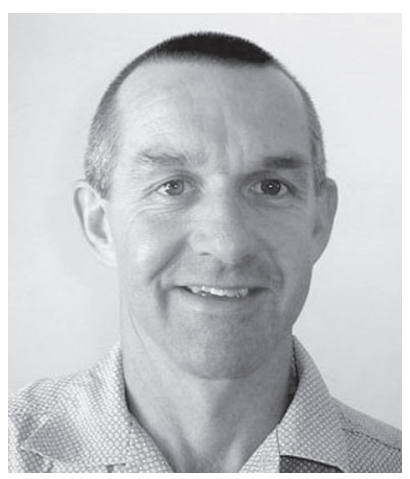

We are in the midst of an exponential growth of access to information. In particular, the development of the Internet has been paralleled by the ease of setting up a blog which assumes some status of authority among the readers and contributors. Now anyone who has access to a computer and the Internet can write on a topic and contribute their viewpoint to a discussion. Apart from the censure of bad or inflammatory language, viewpoints can be displayed freely. This free communication has many advantages and perhaps helps to contribute to the acceptance of a wider range of ideas and tolerance for varying viewpoints.

However, this proliferation of information has introduced new challenges to us. In the past we could consult printed media and, with a relatively high degree of confidence, accept that the information came from a legitimate source. Now one assumes that at one's peril! Instinctively we have to be more vigilant and interrogate information more carefully. However, I do not see this as a problem, as it encourages us to go back to first principles. As the South African Journal of Sports Medicine is written for health professionals from different disciplines who are attempting to have an evidence-based approach to their work, I thought it was a good idea to revisit some of the first principles which govern our work. An evidence-based approach assumes that the work has a scientific foundation. By definition, a scientific approach is based on logical principles derived from well-controlled experimentation. A feature of this approach is that it should be able to stand up to critical scrutiny. This is how the process of science works, and how knowledge slowly accumulates. To assist with screening the accuracy of new information I would like to suggest a few questions we can ask ourselves as a reflex action whenever we are evaluating new information:

Does the information portrayed in the article sound too good to be true? For example, does the product discussed promise quick improvement in health and fitness without requiring any major lifestyle changes? If this is the case alarm bells should ring. Progress in science works at a snail's pace. It may take a paper a year to get published after it has been accepted for publication by a journal. Often unpublished data are presented at conferences, so these data may take even longer to reach the printed form. Therefore any claims of a 'Eureka-type' finding should be viewed with caution. Our understanding of physiology and biochemistry has evolved to the point where we have passed the stage where there will be sudden breakthroughs in knowledge which will have a sudden, dramatic impact on health and fitness. When exercise science and sports medicine were flourishing as an independent discipline in the early 1980s, it was common for breakthroughs in knowledge which resulted in major effects on health or performance. Popularising the ergogenic effects of carbohydrate supplementation during endurance exercise is one of these examples. Tapering after a period of hard training before competition is another example of knowledge in an area accumulating rapidly and having a significant impact on performance. This does not mean that progress in the acquisition of new knowledge has ended; it just means that steps in progress will be smaller and less noticeable than previously, when the discipline of exercise science and sports medicine was on the steep part of the development curve.

When examining the original data described in the article, are the research subjects involved in the experiment the same type of subjects that the data are likely to be applied to? This may sound like a rather logical assumption but it has been violated several times previously. For example, some of the early studies on protein supplementation were done on malnourished individuals. Of course these individuals would flourish after receiving a protein supplement, in contrast to the results one would observe in a well-nourished and healthy person. This however did not prevent the manufacturers of the supplement from capitalising on the results and marketing the product in a healthy population. There are many other examples in the fitness industry where untrained people are shown to have dramatic changes after being exposed to a gimmicky training programme. It stretches the believability factor to assume that highly trained individuals will respond to the same training stimulus in the same way. Nevertheless this trick has been used successfully in the past and made some businesses large amounts of money.

Are there dogmatic statements in the conclusions of the paper? Anyone involved in science will know that there are risks associated with being dogmatic about data! As a student I was taught by one of the top exercise scientists in the world that in the future $50 \%$ of our current knowledge will be shown to be incorrect. He continued with a wry smile by saying that unfortunately we don't know which $50 \%$ it will be! Therefore treat anyone with a dogmatic belief with caution. They clearly have something to gain from their beliefs - either financial or ego!

Therefore, the skills needed by someone trying to critically evaluate a contemporary topic should be refined and developed. Access to information is no longer the limiting factor. Synthesising the information is now the distinguishing factor between someone who has a true understanding and ability to apply the information in an evidence-based way, compared with someone who attempts, but fails to apply their knowledge in an evidence-based way.

We welcome the reader to the first edition of 2009! The goal of this journal is to contribute to the dissemination of information. All papers are peer-reviewed and are required to fulfill requirements which contribute to this goal. This is the first edition in which we have implemented the new guidelines for authors (page 31). We are committed to ensure that the information is communicated in a responsible way. We encourage all the scientists and practitioners involved in health and fitness to contribute to this goal by submitting a paper which may make a contribution. In the past the ability to contribute to the South African Journal of Sports Medicine may have been biased towards academics and scientists. However, we have introduced two new categories (Commentary and Case Studies) which will cater for practitioners who may not be in a position to conduct a formal study. Much of our current knowledge has been ignited by case studies which are sometimes the catalyst for further investigation in a more systematic manner. Just think how gratifying it will be for the author if in the future a research paper or textbook is written on a topic which at present is undefined, but described in a case study published in the South African Journal of Sports Medicine!

\section{Mike Lambert}

Editor-in-Chief 